investigate whether there was a sex difference in change in $\mathrm{FEV}_{1}$ and BMI between 2008-2013, and if this difference could be explained by chronic Pseudomonas aeruginosa (cPsA) infection or CF-related diabetes (CFRD).

Methods Longitudinal analyses (2008-2013) compared male/ female age at cPsA acquisition as well as $\mathrm{FEV}_{1}$ and BMI differences between individuals with cPsA infection. Regression analysis examined for a difference in change in BMI and $\mathrm{FEV}_{1}$ between the sexes depending on CFRD and cPsA status, adjusting for age, genotype and ethnicity. A survival analysis completed the sex comparison.

Results Females were significantly younger than males at the time of new cPsA infection (20.9 vs 22.4 years; $\mathrm{p}<0.001)$ with a lower mean BMI with new cPsA (21.3 vs 22.2 years; $\mathrm{p}<0.001$ ) but no difference in $\mathrm{FEV}_{1}$ at time of new cPsA. Females had greater decline in $\mathrm{FEV}_{1}$ than males $(8.2 \%$ vs $7.0 \%$ over 5 years; $\mathrm{p}<0.001)$, this was even greater in individuals with cPsA $(10.2 \%$ vs $8.2 \%$ in males: $p=0.002)$. Females had less of an increase in BMI than males (0.2 vs 0.6 in males; $<<0.001)$, this difference was only seen in individuals with cPsA. Sex differences in change in BMI were also seen in the CFRD population. Overall, median survival for females was significantly less than males $(39.5$ vs 44.2 years, $\mathrm{p}<0.001)$. Females with CFRD had the worst survival overall. Males without cPsA had the greatest median survival while males with cPsA had similar survival to females irrespective of their cPsA status.

Conclusions Females had earlier cPsA infection and lower BMI. CPsA was associated with greater decline in $\mathrm{FEV}_{1}$ and BMI in females than males, with worse survival in females with cPsA that was not seen in males with cPsA. CFRD was associated with less BMI increase in females, with females with CFRD having worse survival overall. These data suggest a measurable sex difference in clinically relevant CF outcomes in the UK population.

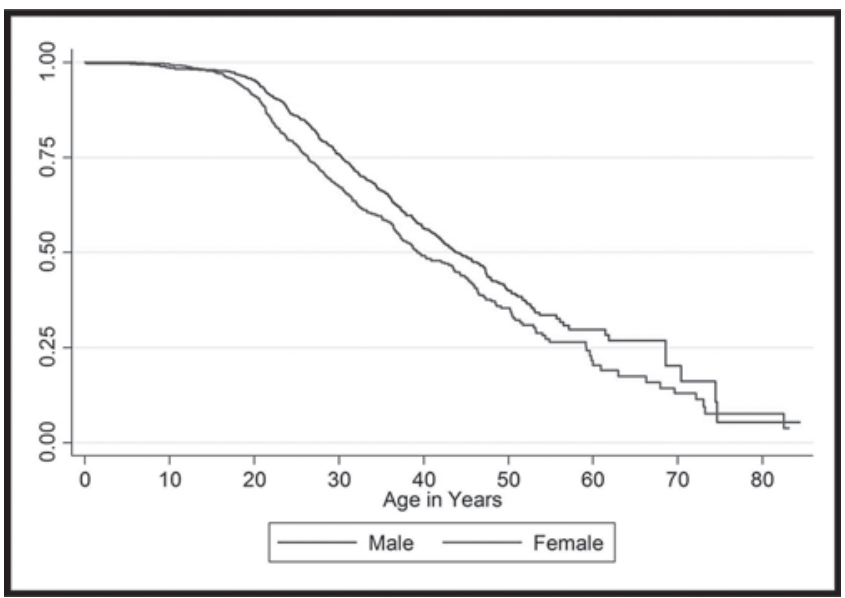

Abstract T4 Figure 1 Kaplan-Meier survival estimates (males and females).

\section{T5 COMPLEMENT PROTEIN C5A INDUCES PROLONGED NEUTROPHIL DYSFUNCTION IN A CLINICALLY RELEVANT MODEL OF HUMAN BACTERAEMIA}

${ }^{1} \mathrm{AJT}$ Wood, ${ }^{1} \mathrm{~A}$ Vassallo, ${ }^{2} \mathrm{~K}$ Okkenhaug, ${ }^{3} \mathrm{~J}$ Scott, ${ }^{3} \mathrm{~J}$ Simpson, ${ }^{1} \mathrm{C}$ Summers, ${ }^{1} \mathrm{ER}$ Chilvers, ${ }^{1}$ A Conway-Morris. ${ }^{1}$ Department of Medicine, University of Cambridge, Cambridge, UK; ${ }^{2}$ Signalling Programme, Babraham Institute, Cambridge, UK; ${ }^{3}$ Institute of Cellular Medicine, Newcastle University, Newcastle, UK

\subsection{6/thoraxjnl-2017-210983.5}

Introduction Critically ill patients are highly susceptible to nosocomial infections including ventilator-associated pneumonia. Susceptibility to nosocomial infection is heavily influenced by immune-cell failure; however the mechanisms underpinning this process remain incompletely understood. ${ }^{1}$ Previously, we have demonstrated that the complement split product C5a impairs the phagocytosis of zymosan by healthy donor and patient neutrophils. ${ }^{2}$ In this study, we investigated the underlying mechanism, duration and preventability of C5a-induced neutrophil dysfunction in a clinically relevant in vitro model.

Methods In a new assay, which permits rapid interrogation of neutrophil functions in whole blood, healthy human or murine blood samples were exposed to C5a or vehicle control, prior to addition of $\mathrm{pH}$-sensitive Staphylococcus aureus bioparticles. Phagocytosis was quantified by flow cytometry with an Attune Next Acoustic Focusing Cytometer (Life Technologies, Paisley, UK). Selective small molecule inhibitors, alternative pro-inflammatory agents, and neutrophils from knock-out mice were used to address our experimental questions.

Results C5a rapidly reduced neutrophil phagocytosis of Staphylococcus aureus in human whole blood by $39.5 \%$ $(\mathrm{p}<0.0001)$. Moreover, this phagocytic impairment increased over time post-exposure. In contrast to C5a, LPS and platelet activating factor (PAF) increased phagocytosis $(p<0.01$ and $\mathrm{p}<0.05$ respectively). Prior phagocytosis protected neutrophils from subsequent C5a-induced phagocytic impairment, but this was not recapitulated by exposing neutrophils to soluble priming agents. C5a-induced phagocytic impairment was PI3-kinase dependent in isolated neutrophils, but not in human and murine whole blood.

Conclusions This is the first study to demonstrate the selective ability of C5a to impair neutrophil phagocytosis of a clinically relevant pathogen in a whole blood model which mimics bacteraemia. It also provides intriguing insights into the potential mechanisms by which this may occur, including the temporal dependence of C5a and bacterial exposure. Finally, the ability of PI3-kinase inhibition to ameliorate neutrophil dysfunction may vary depending on whether inhibitors are administered topically or systemically.

\section{REFERENCES}

1. Conway-Morris A, et al. Br J Anaesth 2013;111:778-87.

2. Conway-Morris A, et al. Blood 2011;117:5178-88. 